\title{
Erratum to: Vitamin D deficiency in patients with either rheumatic diseases or inflammatory bowel diseases on biologic therapy
}

Vincenzo Bruzzese $^{1} \cdot$ Angelo Zullo $^{1}$ - Andrea Picchianti Diamanti ${ }^{2}$.

Lorenzo Ridola $^{3}$ - Roberto Lorenzetti ${ }^{1}$ - Cinzia Marrese ${ }^{1}$ - Palma Scolieri ${ }^{1}$.

Vincenzo De Francesco $^{4}$. Cesare Hassan ${ }^{1} \cdot$ Alberto Migliore $^{5} \cdot$ Bruno Laganà $^{2}$

Published online: 19 May 2016

(C) SIMI 2016

\section{Erratum to: Intern Emerg Med}

DOI 10.1007/s11739-016-1415-9

In the original publication, the third author's name was incorrectly published as Andrea Piacchianti Diamanti. The correct name should read as "Andrea Picchianti Diamanti".

The online version of the original article can be found under doi:10.1007/s11739-016-1415-9.

Angelo Zullo

angelozullo66@yahoo.it

Vincenzo Bruzzese

vinbruzzese@tiscali.it

1 Medicina Interna, Reumatologia e Gastroenterologia,

Ospedale Nuovo Regina Margherita, Via E. Morosini, 30,

00153 Rome, Italy

2 Allergy, Clinical Immunology and Rheumatology,

'Sapienza' University of Rome, S. Andrea University

Hospital, Rome, Italy

3 Gastroenterology Unit, Sapienza University of Rome "Polo

Pontino" Hospital, Latina, Italy

4 Gastroenteroolgy Unit, 'RiUniti' Hospital, Foggia, Italy

5 Rheumatology Unit, San Pietro Fatebenefratelli Hospital, Rome, Italy 